\title{
WhatsApp como canal de información política en España: credibilidad, perfil de usuarios y compartición de contenidos
}

\section{WhatsApp as a political information channel in Spain: credibility, user profile, and content sharing}

\author{
Carles Pont-Sorribes; Reinald Besalú; Lluís Codina
}

Cómo citar este artículo:

Pont-Sorribes, Carles; Besalú, Reinald; Codina, Lluís (2020). “WhatsApp como canal de información política en España: credibilidad, perfil de usuarios y compartición de contenidos". Profesional de la información, v. 29, n. 6, e290619.

https://doi.org/10.3145/epi.2020.nov.19

Artículo recibido el 20-05-2020

Aceptación definitiva: 29-06-2020

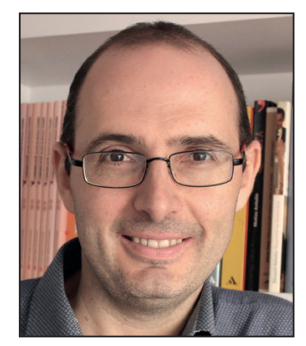

Carles Pont-Sorribes

https://orcid.org/0000-0003-1135-4245

Universitat Pompeu Fabra

Departament de Comunicació

Roc Boronat, 138

08018 Barcelona, España

carles.pont@upf.edu

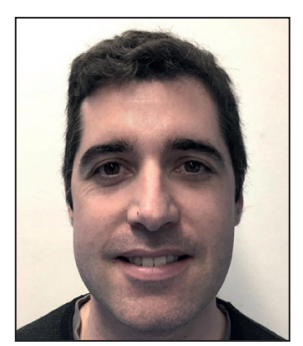

Reinald Besalú

https://orcid.org/0000-0002-2485-361X

Universitat Pompeu Fabra

Departament de Comunicació

Roc Boronat, 138

08018 Barcelona, España

reinald.besalu@upf.edu

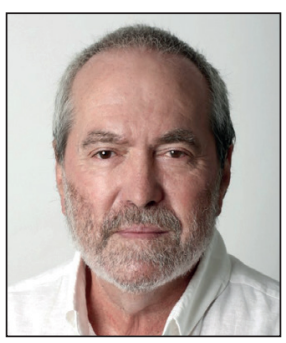
Lluís Codina
https://orcid.org/0000-0001-7020-1631
Universitat Pompeu Fabra
Departament de Comunicació
Roc Boronat, 138. 08018 Barcelona, España
UPF Barcelona School of Management
Balmes, 134, 08008 Barcelona, España
Iluis.codina@upf.edu

\section{Resumen}

La mensajería instantánea móvil ha cambiado nuestros hábitos comunicativos, convirtiéndose no sólo en el principal canal de comunicación mediante dispositivos electrónicos, sino también en un nuevo canal a través del que informarse de política y con el que interactuar con los contenidos informativos políticos. Por ello, los objetivos principales de esta investigación son: a) identificar la utilidad que los españoles dan a la plataforma WhatsApp como canal de información política; b) conocer las prácticas de los españoles ante la información política recibida en la plataforma; y c) analizar si la información política es compartida o no en los grupos de la aplicación, así como el grado de credibilidad que le otorgan los ciudadanos. La investigación emplea una metodología cuantitativa centrada en el diseño de una encuesta online con una muestra de 1.669 ciudadanos representativa de la población española, realizada por la empresa de demoscopia YouGov y ponderada por sexo, edad y zona de residencia. La investigación concluye que la mensajería instantánea se ha instalado de forma clara en el ecosistema comunicativo español. Un 64\% de los españoles recibe al menos una noticia semanal de actualidad política a través de WhatsApp y un $24 \%$ recibe 5 o más noticias por semana. 


\title{
Palabras clave
}

Información política; Comunicación política; Periodismo; Noticias falsas; Fake news; Credibilidad; Compartición de contenido; Mensajería instantánea; WhatsApp.

\begin{abstract}
Mobile instant messaging has changed our communication habits. It has become the main communication channel between people through electronic devices and a new channel that can provide information about politics as well as allow interaction with it. For this reason, the main objectives of this research are: (a) to identify how Spanish citizens use WhatsApp as a political information tool, (b) to understand how Spanish citizens react to political information received via this platform, and (c) to analyze whether political information is shared in groups on the application and evaluate its level of credibility. A quantitative methodology is used in this research through the design of an online survey with a sample of 1,669 representative Spanish citizens, realized by the demographic studies company YouGov and weighted by sex, age, and area of residence. The study concludes that instant messaging has become clearly established in the Spanish communicative ecosystem, with $64 \%$ of Spanish people receiving at least one piece of news about political issues weekly through WhatsApp and $24 \%$ receiving five or more per week.
\end{abstract}

\section{Keywords}

Political information; Political communication; Journalism; Fake news; Credibility; Content sharing; Instant messaging; WhatsApp.

\section{Introducción}

La mensajería instantánea móvil se ha convertido en un canal comunicativo masivo. Plataformas como WhatsApp, Telegram, Facebook Messenger o HangOuts, entre otras, conectan de forma directa a millones de personas de todo el mundo. Este artículo se centra en el estudio de WhatsApp, una de las plataformas más utilizadas y la que tiene mayor incidencia en comunicación social (Newman et al., 2020). Fue fundada por Jan Koum e irrumpió en el entorno digital en 2009 como un servicio gratuito de mensajería instantánea para teléfonos inteligentes.

WhatsApp ofrece tres formas de comunicación:

- se puede mantener una conversación directa con otra persona;

- se mantienen conversaciones a partir de listas de difusión;

- facilita la creación de grupos de usuarios con mecanismos de conversación grupal, que son utilizados a menudo para la viralización de contenidos.

La aplicación utiliza plenamente las características más importantes de los teléfonos inteligentes, ya que permite una comunicación sencilla y directa, de tipo asincrónico o interactiva, mediante mensajes de texto o voz entre dos o más personas, y su propósito es fundamentalmente mantener a las personas comunicadas pese a la distancia (Besalú et al., 2019).

En los últimos años el ecosistema informativo digital ha cambiado nuestros hábitos comunicativos de una forma acelerada (Jenkins, 2008; Chadwick, 2013; Casero-Ripollés; Feenstra; Tormey, 2016). Esta innovación ha transformado las comunicaciones personales de modo vertiginoso. Una pregunta que cabe plantearse es si también han cambiado la obtención y la credibilidad de la información pública y política que se recibe a diario. Las empresas, los grupos de amigos, pero también las formaciones políticas o las administraciones públicas utilizan WhatsApp para organizarse, informar, difundir comunicados o simplemente interactuar con grupos de militantes o simpatizantes para mandar mensajes persuasivos o pedir el voto, llegando a miles de ciudadanos.

Diversas investigaciones demuestran que los ciudadanos utilizan los dispositivos móviles como el primer medio de acceso a internet en España, lo que supone un cambio en la forma en que el público se informa e interactúa con los contenidos informativos (Varona-Aramburu; Sánchez-Martín; Arrocha, 2017). Incluso muchos estudiosos de la democracia digital señalan el potencial de internet y los móviles inteligentes para la comunicación democrática entre los ciudadanos. Yan, Sivakumar y Xenos sugieren que:

"la discusión deliberativa coexiste con formas liberales de comunicación individualista y comunitaria en foros online" (Yan; Sivakumar; Xenos, 2018).

Por otra parte, autores como Boczek y Koppers, (2020) demuestran que WhatsApp se ha convertido en un canal informativo relevante. Estos autores estudian su uso por parte de las redacciones de diarios de Alemania, observando si este es tan habitual como el de las redes sociales Facebook, Instagram y Twitter. El análisis se basa en 3.745 mensajes enviados a canales de WhatsApp de medios de comunicación y en una encuesta online a periodistas que trabajan con redes sociales. Según el estudio

"los canales de WhatsApp permiten a los medios de comunicación estar más cerca de su audiencia gracias a su tecnología innovadora y un nuevo tipo de relación que crea más engagement". 
Si las personas reducen su consumo de medios tradicionales o de una red social, la difusión de noticias por WhatsApp suele ser una alternativa frecuente. La visión del periodista se centra en ver el público como un usuario activo y no como un socio que colabora en la elaboración de la noticia.

Otra investigación relevante sobre el uso informativo es la de Dodds (2019), que examina los incentivos que llevan a los periodistas a usar WhatsApp y las consecuencias que esta aplicación ha tenido en las prácticas de información de periodistas de dos redacciones chilenas. El estudio sugiere que su utilización ha afectado la relación entre periodistas y fuentes tanto a nivel personal como profesional y que es la aplicación más utilizada por fuentes y periodistas para compartir información. También se concluye que ayuda a privilegiar las relaciones virtuales sobre las presenciales. Palomo y Sedano (2018) en su estudio sobre la creación de la sección B de Bulo en el diario Sur (Málaga), su funcionamiento y su repercusión en redes sociales, concluyen que el uso de programas de fact-checking por parte de los diarios permite reconstruir el vínculo de confianza entre medio de comunicación y ciudadano y señalan que WhatsApp se adapta bien a las necesidades de los medios pequeños o locales.

El estudio que aquí se presenta tiene tres objetivos principales:

a) identificar la utilidad que dan los españoles a WhatsApp;

b) conocer sus prácticas ante la información política recibida a su través;

c) analizar si la información política es compartida o no en los grupos y el nivel de credibilidad que le otorgan los ciudadanos.

La investigación del comportamiento de los ciudadanos en WhatsApp no es sencilla y los investigadores sociales tienen que recurrir a estudios de recepción para conocer la utilización e interacción con la plataforma, dado que es de uso privado. En consecuencia, la manera más eficiente y homologable científicamente es el uso de encuestas o focus groups. Esta investigación se ha llevado a cabo con el soporte de analistas de la empresa de estudios de mercado YouGov Spain.

\section{Marco teórico y contexto}

La irrupción de WhatsApp hizo proliferar los estudios científicos sobre su uso en diferentes contextos. Sirvan como ejemplo: Fletcher y Park (2017); Swart, Peters y Broersma (2019); Sedano y Palomo (2018); Gutiérrez-Rubí (2015); y Quiroz-Pacheco (2015) entre otros. Estas aportaciones son relevantes en el contexto actual porque permiten observar el entramado que representa no sólo WhatsApp como aplicación, sino también como parte del proceso informativo y comunicativo de la política. Las investigaciones sobre WhatsApp tienen su importancia desde múltiples perspectivas:

- como medio de comunicación e información;

- como sistema de mensajería que ha modificado el lenguaje virtual, instantáneo, dentro de la categoría de lo hablado-escrito (Calero-Vaquera, 2014; Giraldo-Giraldo; Ríos-Londoño; Cardona-Cifuentes, 2018);

- como canal que ha modificado los hábitos del periodismo (Fares, 2018; Boczek; Koppers, 2020); Dodds (2019).

En este último punto es importante destacar que WhatsApp ha hecho aún más evidente la necesidad de verificar las fuentes y el rigor en la información -noticias falsas o fake news- (Palomo; Sedano, 2018), que se han convertido en la actualidad en uno de los principales problemas del entorno digital. Una aportación relevante sobre el uso de WhatsApp como plataforma comunicativa es la de Gil de Zúñiga, Ardèvol-Abreu y Casero-Ripollés (2019), que concluye que

"WhatsApp proporciona un entorno más íntimo y controlado en el que los usuarios pueden reunir y compartir noticias casi simultáneamente, discutir políticas y movilizar a otros".

Ese estudio revela además que la discusión en esta plataforma tiene una influencia positiva en el activismo y un efecto más matizado en la participación convencional. Valeriani y Vaccari (2018) también aportan conclusiones en esa línea, en este caso con un estudio comparativo de Alemania, Italia y Reino Unido. Los autores demuestran que los encuestados que usan mensajería instantánea la emplean para publicar mensajes políticos y discutir sobre política. También concluyen que

"las conversaciones políticas sobre mensajería se asocian positivamente con la tendencia de los usuarios a censurarse políticamente en los sitios de redes sociales y, en menor medida, con el extremismo ideológico".

Un aspecto importante de este artículo es conocer el índice de credibilidad o confianza que los ciudadanos otorgan a WhatsApp. Para dejar claro que hablamos de términos semejantes, aunque no exactamente iguales, nos remitimos al trabajo de Go et al. (2016) en el que plantean una interesante reflexión sobre la relación entre credibilidad y confianza (trust). Según estos autores, si por un lado la tradición de la media psychology suele centrarse en el concepto de credibilidad como una percepción individual, la tradición de la media sociology es más proclive a emplear el término "confianza" como capital social. En todo caso, tal y como también advierte Tsfati (2010), la credibilidad debería entenderse como uno de los indicadores que permiten evaluar la confianza en los medios de comunicación a nivel social. En este trabajo utilizaremos indistintamente ambos términos a partir de una visión amplia e interdisciplinar de estos conceptos.

En cuanto a la confianza, destacamos la investigación de Fletcher y Park (2017), quienes empleando los datos de la encuesta del Reuters Institute, Digital news report ( $\mathrm{N}=21.524)$, exploraron la confianza en los medios de comunicación en función de las fuentes que se utilizan y la participación en las noticias online. La muestra recoge datos de 11 países, con 
resultados que muestran que las personas con niveles bajos de confianza en los medios suelen preferir fuentes de información que no provienen de medios mainstream. Estos ciudadanos utilizan las redes sociales y los blogs para informarse y son más proclives a participar online comentando noticias de actualidad. Del mismo modo, un experimento con 364 estudiantes universitarios de Turcotte et al. (2015) nos permite conocer la confianza mediática. El estudio demuestra que el hecho de que un amigo haya recomendado socialmente un medio de comunicación genera más posibilidades de que el individuo quiera seguir consultando noticias de este medio. A la vez, tiene un efecto positivo en los niveles de confianza entre medio e individuo y sugiere que las noticias en las que se confía guían el comportamiento. Se demuestra que la comunicación interpersonal importa en la credibilidad de las noticias y que el liderazgo percibido refuerza esta credibilidad. En una línea similar, Ardèvol-Abreu y Gil de Zúñiga (2017) analizan los niveles de confianza de los encuestados en noticias de plataformas de medios de comunicación. Los investigadores demuestran que la confianza en los medios no tiene relación con el consumo de un tipo de medios: tradicionales, ciudadanos o de redes sociales. También concluyen que cuando el individuo percibe que una noticia es presentada a través de un sesgo ideológico, esto tiene un efecto negativo generalizado en el uso de medios de comunicación, independientemente del tipo de medio. Es posible que el sesgo percibido traslade los ciudadanos hacia fuentes de información diferentes, alternativas, que consideren menos sesgadas.

En otra investigación destacable, Swart, Peters y Broersma (2019) analizan los grupos privados de redes sociales y sus conversaciones a partir de seis focus groups formados por personas que ya tenían un vínculo personal. Entre estos vínculos se encuentra alguna variable social compartida como el lugar de vivienda, la comunidad laboral o el ocio. La investigación quiere demostrar qué papel tienen las estructuras relacionales en la interacción con las noticias de las redes sociales. Los autores concluyen que en función del objetivo del grupo hay ciertas discusiones que están permitidas o no. Por ejemplo, el grupo de WhatsApp de maestros de una escuela no sirve para debatir sobre noticias de actualidad. Si las noticias se perciben como un tema seguro para la conversación, sí que se espera que los miembros del grupo las discutan o las lean pasivamente. En función de las normas y el propósito designado del grupo privado se darán ciertas discusiones o no. Además se concluye que el individuo sigue diferentes formas de interacción en función del grupo privado al cual se dirige.

Una investigación de Valenzuela, Bachmann y Bargsted (2019) examina las prácticas de compartición de información de los usuarios de WhatsApp, haciendo una comparativa de los efectos de difusión de contenido personal y de asuntos públicos. El artículo demuestra un incremento del uso de la plataforma por varios motivos, no únicamente para compartir noticias, sino más bien por aspectos de la propia relación. Los autores concluyen que hay una fuerte correlación entre el uso de WhatsApp para compartir contenido político y su participación en protestas políticas, pero no tiene por qué ser indicador de la acción de voto.

Por otra parte, Elishar-Malka, Ariel y Avidar (2015), estudian el papel de WhatsApp en ciudadanos que estuvieron expuestos a amenazas de guerra durante julio de 2014, y sugieren que la plataforma desempeñó un papel multifuncional central en la vida de sus usuarios durante el tiempo de guerra: "funcionando como un canal de comunicación masivo e interpersonal". La investigación demuestra que utilizaron la aplicación diariamente para diversos fines:

"obtener noticias y actualizaciones sobre la guerra; saber de sus seres queridos; publicar mensajes satíricos humorísticos; difundir rumores relacionados con la guerra; y promover acciones de ayuda voluntaria".

Sin embargo, en el ámbito de la desinformación y las fake news, WhatsApp ha centrado buena parte de las investigaciones de manera directa o indirecta en varios países. Así, un estudio de Resende et al. (2019) demuestra que en eventos sociales en Brasil los grupos de WhatsApp fueron fuente de propagación de noticias falsas. De igual forma, el trabajo de Bernal-Triviño y Clares-Gavilán (2019) señala que la difusión viral de información en redes sociales, el móvil y los servicios de mensajería favorece compartir noticias falsas y sin verificar, asegurando que España es uno de los países europeos que encabeza la recepción de este tipo de contenidos.

Otra aportación relevante sobre participación política es la de Vaccari y Valeriani, (2018). Este trabajo trata sobre la doble proyección política, es decir, sobre la relación entre ver contenidos políticos en la televisión mientras los leemos y comentamos en las redes sociales. La investigación abarca ocho democracias occidentales: Dinamarca, Alemania, Grecia, Italia, Polonia, España, Reino Unido y Estados Unidos, y sugiere que hay una correlación positiva entre la frecuencia con la que los ciudadanos hacen doble pantalla con el contenido político y sus niveles generales de participación. Dicha correlación es más fuerte entre los encuestados con menor nivel de interés en la política, y la participación también es significativamente más fuerte en los países cuyos sistemas de medios cuentan con las emisoras de servicio público más sólidas.

Finalmente, Casero-Ripollés (2018) sugiere que los nuevos medios están trayendo consigo un nuevo entorno comunicativo más híbrido y una lógica basada en nuevos principios y criterios de funcionamiento que afectan a la totalidad del proceso informativo.

Por todo ello la investigación que aquí se presenta tiene como objetivo conocer el uso, pero también la credibilidad, que los ciudadanos españoles dan a WhatsApp para informarse de política. 
Para el desarrollo de este estudio se plantearon las siguientes hipótesis:

a) WhatsApp es utilizado por los ciudadanos para informarse sobre la actualidad política;

b) los españoles comparten mayoritariamente la información que reciben a través de WhatsApp;

b1) en WhatsApp existe una mayor propensión de los ciudadanos a compartir las informaciones acompañadas de material audiovisual;

c) los ciudadanos otorgan una credibilidad baja a las noticias que reciben por mensajería instantánea;

c1) los ciudadanos de menor edad tienden a otorgar más credibilidad a las informaciones que reciben a través de WhatsApp;

d) los españoles suelen comentar la actualidad política a través de WhatsApp.

\section{Metodología}

La investigación emplea una metodología cuantitativa centrada en una encuesta online con una muestra representativa de la población española, realizada por la empresa de demoscopia YouGov a partir del diseño de las preguntas de los investigadores del estudio. Para la recogida de información se empleó una escala Likert de 1-5 en la que 1 corresponde al indicador más bajo de medida (Nunca y Ninguna credibilidad según el caso); y 5 al indicador mayor (Muy a menudo, Total credibilidad). La recolección de datos se realizó en febrero de 2019 y la aplicación de cada cuestionario se hizo de forma individual, con un tiempo estimado para responder la encuesta entre 15 y 20 minutos. La muestra con la que se trabajó estuvo compuesta por 1.669 ciudadanos mayores de edad, representativos del conjunto de la población española y ponderados a nivel de sexo, edad y zona de residencia (Nielsen areas). La muestra está conformada por un $49,6 \%$ de hombres $(N=827)$ y un $50,4 \%$ de mujeres $(N=842)$. Por edades, un $8,6 \%$ tienen entre 18 y 24 años ( $N=144)$, un $15,6 \%$ entre 25 y 34 años ( $N=261)$, un $21,4 \%$ entre 35 y 44 años ( $N=357)$, un 19,4\% entre 45 y 54 años ( $N=324)$ y un $34,9 \%$ tienen 55 años o más ( $N=583)$. Finalmente, respecto a la zona de residencia (Nielsen areas), un $21,7 \%$ residen en el noreste ( $N=362)$, un $14,2 \%$ en levante $(N=237)$, un $23 \%$ en el sur $(N=384)$, un $22,4 \%$ en el centro $(N=374)$, un $9,5 \%$ en el noroeste $(\mathrm{N}=159)$ y un $9,2 \%$ en el norte $(\mathrm{N}=153)$. Para un nivel de confianza del $95 \%$ y $\mathrm{p}=\mathrm{q}=0.5$, el error muestral global es del $+/-2,4 \%$.

\section{Resultados}

\subsection{WhatsApp como fuente informativa}

En el estudio se pregunta sobre el número de noticias de actualidad política que recibieron los ciudadanos a través de WhatsApp durante la última semana con un intervalo de respuesta de: "ninguna; una; entre 2 y 4 ; entre 5 y 10; y más de 10 noticias". Los resultados demuestran que el $64 \%$ de los ciudadanos españoles ha recibido como mínimo una noticia de actualidad política durante la última semana mediante esta aplicación. La mayoría de los encuestados leyeron entre dos y cuatro noticias, mientras que el $36 \%$ dice no haber recibido ninguna información a través de la aplicación los últimos siete días. Además, se puede observar una ligera asociación positiva entre la edad de los encuestados y el número de noticias de actualidad política recibidas; es decir, que los mayores tienden a recibir más noticias que los más jóvenes $\left(\chi^{2}[16, N=1652]=67,535, p=0.000 ; d\right.$ de Somers=0.069, $\left.p=0.000\right)$. El número de personas que no reciben noticias de actualidad política en la plataforma no varía de forma significativa entre las distintas edades, pero sí existen diferencias notables por edad entre los que reciben semanalmente más de 10 noticias, que son menos del 5\% de los ciudadanos menores de 45 años y prácticamente el $12 \%$ de los ciudadanos de 45 años y más.

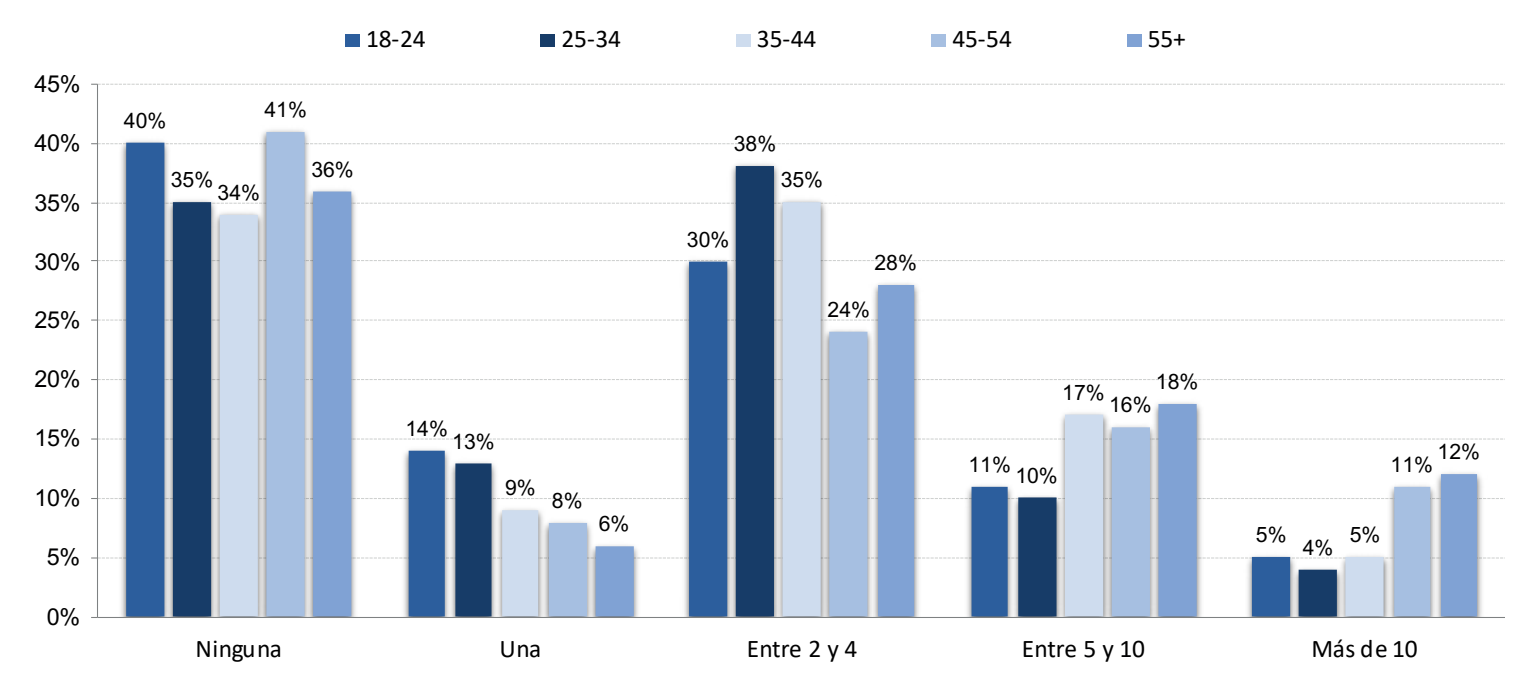

Gráfico 1. Número de noticias sobre actualidad política recibidas a través de WhatsApp durante la última semana por edad 
Por otra parte, existen diferencias estadísticamente significativas entre hombres y mujeres en esta cuestión $\left(\chi^{2}[4, N=1652]=26,405, p=0.000 ;\right.$ Coeficiente de Incertidumbre $=0.006, p=0.000$ ). El $68 \%$ de los hombres ha recibido alguna información política en la última semana, porcentaje que se reduce al $59 \%$ entre las mujeres. Además, el $29 \%$ de los hombres recibe semanalmente 5 o más noticias, porcentaje que en el caso de las mujeres desciende al $20 \%$.

Estos resultados confirman parte de la hipótesis inicial que plantea que WhatsApp es utilizado por los ciudadanos para recibir e informarse sobre la actualidad política.

\subsection{Compartir y comentar noticias políticas}

Uno de los retos importantes de la investigación es conocer si además de informarse de cuestiones políticas, los españoles comparten esta información entre sus contactos. En consecuencia, se preguntó si los ciudadanos utilizaban WhatsApp para compartir noticias. Empleando la escala de respuesta Likert de 1 - 5, en la que 1 corresponde a "nunca" y 5 "muy a menudo", los encuestados respondieron que utilizan WhatsApp para compartir noticias en un 77\% de los casos, mientras que un $23 \%$ utiliza la plataforma pero no comparte nunca las noticias, por lo que también queda confirmada la hipótesis (b). Además se observa que la predisposición a compartir se puede asociar positivamente con el número de informaciones que se reciben $\left(\chi^{2}[16, N=1652]=596,466, p=0.000\right.$; Gamma $\left.=0.605, p=0.000\right)$. Es decir, en términos generales, cuantas más noticias de actualidad política se reciben, más tendencia a compartirlas. En cambio, no existen diferencias estadísticamente significativas en la tendencia a compartir informaciones por WhatsApp según el género y la edad.

WhatsApp es un canal de comunicación utilizado por todos los segmentos de la población, y los mensajes que recibimos para informarnos sobre la actualidad política pueden pasar por un filtro propio. Se observa que el hecho de que el contenido sea audiovisual (vídeo o fotografía) determina una mayor propensión de los ciudadanos a compartir las informaciones, tal y como se apunta en la hipótesis (b1).

En una escala de 1 a 5, en la que 1 es "nunca" y 5 "muy a menudo", los españoles se sitúan de media en el 2,36 cuando se trata de compartir información por WhatsApp, mientras que la puntuación sube a 2,55 cuando se trata específicamente de informaciones en formato vídeo o fotografía (la propensión media a compartir se incrementa en un $8 \%$ ). Si un $23 \%$ de los encuestados afirma que nunca comparten información por WhatsApp, este porcentaje se reduce al $19 \%$ si se especifica que se trata de información con fotografía o vídeo. De este modo, aunque no puede afirmarse que la propensión a compartir frecuentemente informaciones por la aplicación sea mayoritaria en ninguno de los casos (ya que la mayoría lo hace sólo a veces, sean las informaciones en formato texto o audiovisual), sí se incrementa ligeramente la predisposición media a compartir en el caso de informaciones basadas en fotografías o vídeos.

La tendencia a utilizar la plataforma para comentar la actualidad política es baja entre aproximadamente la mitad de los encuestados (el 52\% "nunca" o "casi nunca" lo hacen), mientras que solo el $17 \%$ lo hace habitualmente o muy a menudo. Aunque no puede refutarse la hipótesis (d), según la cual los españoles tienden a utilizar la plataforma para comentar la actualidad política, esta práctica es habitual sólo entre una minoría.

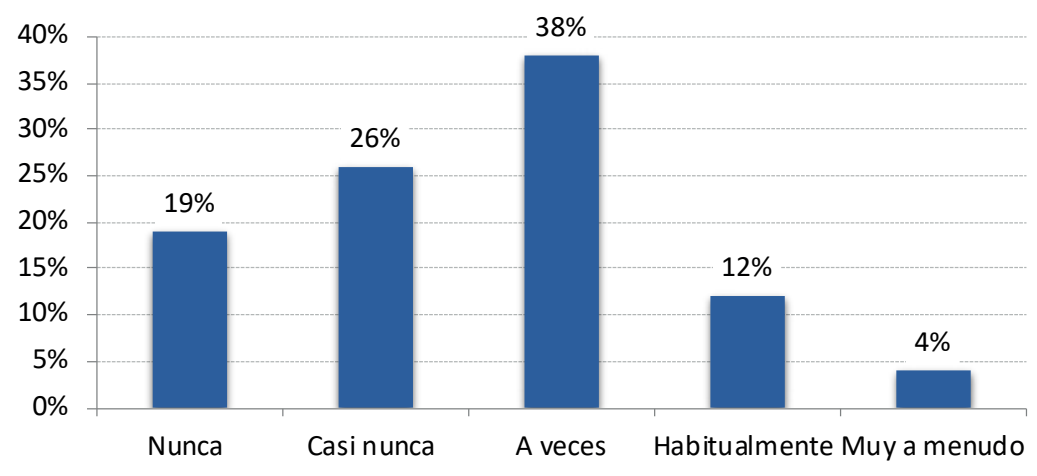

Gráfico 2. Frecuencia con la que se comparten noticias con fotografía/vídeo sobre actualidad política a través de WhatsApp

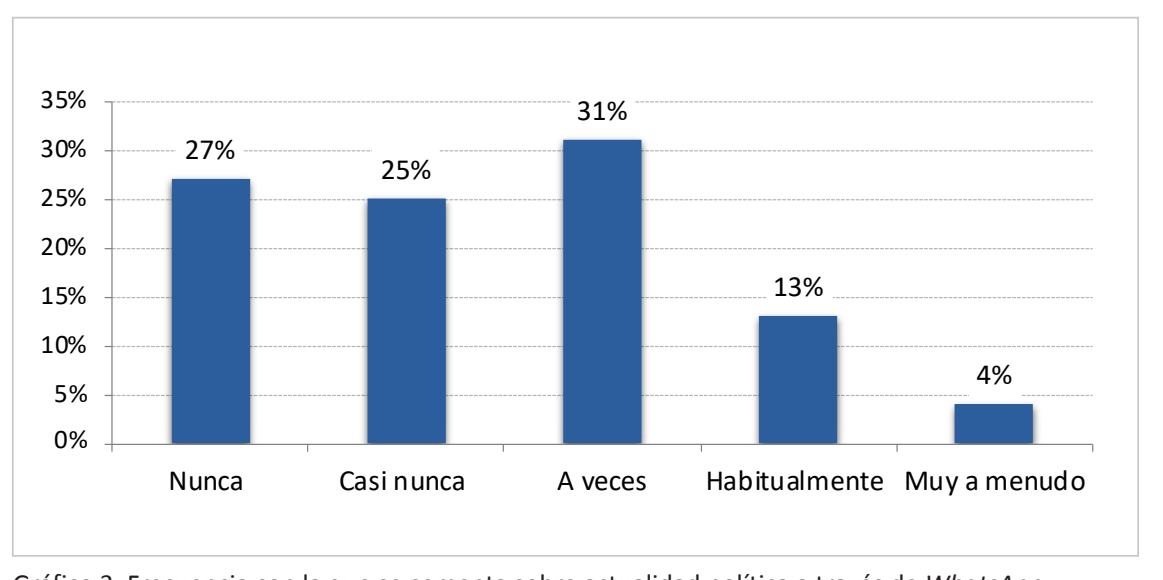

Gráfico 3. Frecuencia con la que se comenta sobre actualidad política a través de WhatsApp 


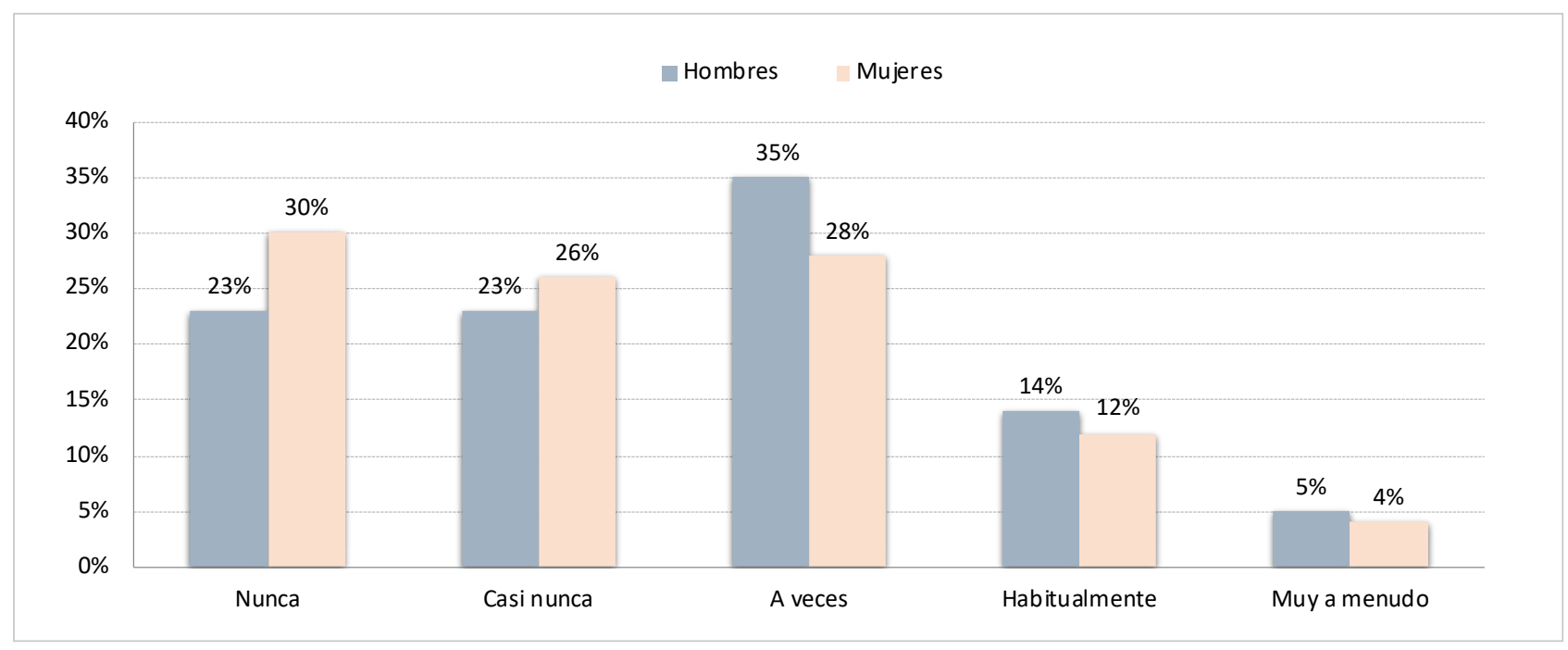

Gráfico 4. Frecuencia con la que se comenta sobre actualidad política a través de WhatsApp, por género

Por otro lado, la predisposición a comentar la actualidad política se asocia positivamente con el número de informaciones que se reciben por WhatsApp $\left(\chi^{2}[16, N=1652]=502,797, p=0.000\right.$; Gamma=0.554, $\left.p=0.000\right)$. Es decir, en términos generales, cuantas más noticias de actualidad política se reciben por esta plataforma, más tendencia a comentar la actualidad política a través de la aplicación. Se encontraron diferencias en la frecuencia con la que se comenta la actualidad política a través de la aplicación entre hombres y mujeres $(F(1,1667)=16.274 ; p=0.000)$. En una escala de 1 a 5 en la que 1 significa nunca y 5 significa muy a menudo, la media de compartición de los hombres fue de 2,55, mientras que la de las mujeres fue de 2,32. Especialmente las diferencias son significativas entre los que afirman que nunca comentan la actualidad política: son el $30 \%$ de las mujeres y solo el $23 \%$ de los hombres. Por otro lado, se observa también cierta asociación con el nivel formativo $(F(12,1656)=2.538 ; p<0.005)$ : los menos formados responden en mayor proporción que nunca comentan la actualidad política a través de WhatsApp.

\subsection{Credibilidad informativa, grupos de discusión y política}

Los mainstream media o medios convencionales, especialmente los audiovisuales, determinaban unas franjas horarias establecidas en las que los ciudadanos tenían un espacio mediático en el cual estaban expuestos a imágenes informativas. Sin embargo, la evolución del espacio mediático al espacio hipermediático ha tenido como consecuencia que el ciudadano tenga acceso a imágenes informativas a través de medios y plataformas sociales constantemente. Este flujo inmediato de contenidos facilita la circulación de noticias falsas o fake news, que son difundidas en muchos casos de forma masiva por los propios usuarios.

En cuanto a la credibilidad que otorgan a las noticias sobre la actualidad política que reciben a través de WhatsApp, sólo el 3\% de los ciudadanos cree totalmente en ellas, mientras que el $97 \%$ duda en mayor o menor grado de su credibilidad. No podemos validar la tercera hipótesis (c), que establecía que los ciudadanos otorgan una credibilidad baja o muy baja a las informaciones que reciben por WhatsApp. Así, en una escala de 1 a 5 en que 1 significa "ninguna credibilidad" $y$ 5 "total credibilidad", los españoles se sitúan de media en el 2,59 (desviación típica: 1,001), y el número de ciudadanos que otorgan una credibilidad baja o muy baja se sitúa por debajo del $50 \%$ (son el $44 \%$ ). Se puede afirmar, por lo tanto, que la credibilidad otorgada a WhatsApp como fuente de información política es en términos generales media, en ningún caso baja. No existen diferencias significativas entre edades ni entre géneros por lo que respecta a

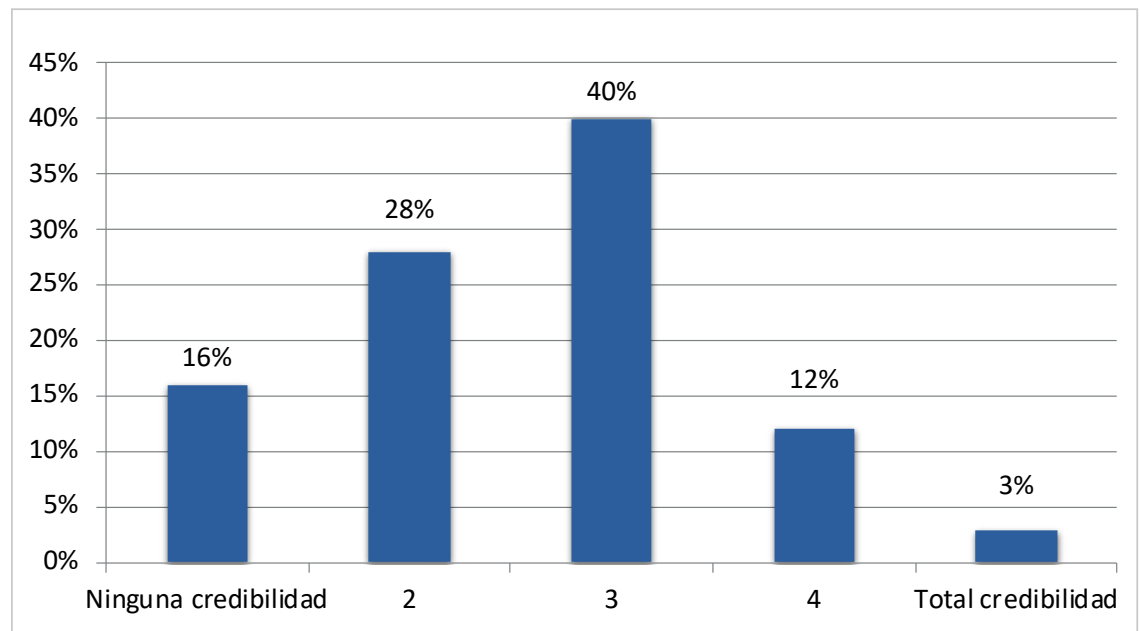
esta cuestión.

Gráfico 5. Credibilidad de las noticias sobre actualidad política recibidas a través de WhatsApp 


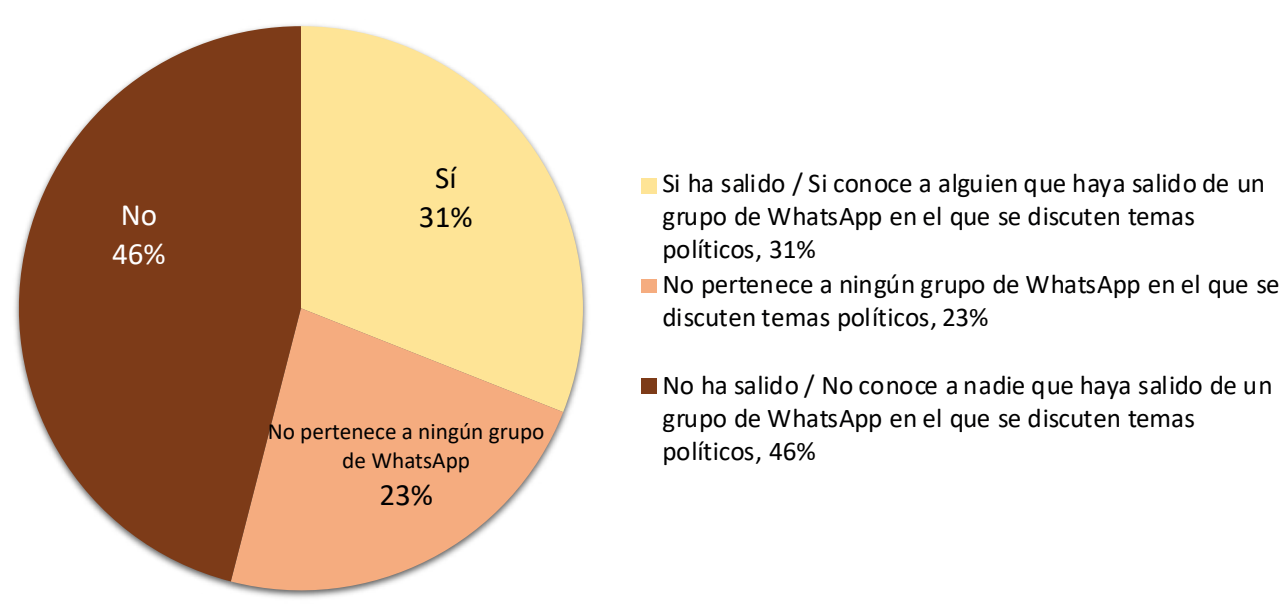

Gráfico 6. Credibilidad de las noticias sobre actualidad política recibidas a través de WhatsApp

En otro orden de cosas, la investigación quería saber si los ciudadanos españoles alguna vez habían estado en algún grupo de WhatsApp del que alguien hubiera salido por motivos políticos -incluyéndose a sí mismos. Las respuestas se limitaron a tres opciones: Si; No; y No pertenezco a ningún grupo de WhatsApp en el que se discutan temas políticos. Los resultados arrojan que el $77 \%$ de los ciudadanos forman parte de al menos un grupo de conversación en la plataforma en el que se habla de política. Por otro lado, se observa que el $23 \%$ de los encuestados señaló que no pertenece a ningún grupo en el que de momento- se discutan temas políticos. Al desagregar el $77 \%$ de los casos en los que se habla de política, podemos afirmar que el $31 \%$ de los ciudadanos pertenece a un grupo que alguien o ellos mismos han abandonado por motivos políticos, mientras que el $46 \%$ restante no ha tenido esta experiencia.

Existen ligeras diferencias estadísticamente significativas entre hombres y mujeres $\left(\chi^{2}[2, N=1669]=\right.$ 22,797, $p=0.000$; Coeficiente de incertidumbre $=0.006, \quad p=0.000$ ). Específicamente, hay más hombres que pertenecen a un grupo que alguien ha abandonado por motivos políticos (el 35\%, frente al $27 \%$ de mujeres), y más mujeres que no pertenecen a ningún grupo de WhatsApp en el que se discutan cuestiones políticas (el $27 \%$, frente al $19 \%$ de hombres).

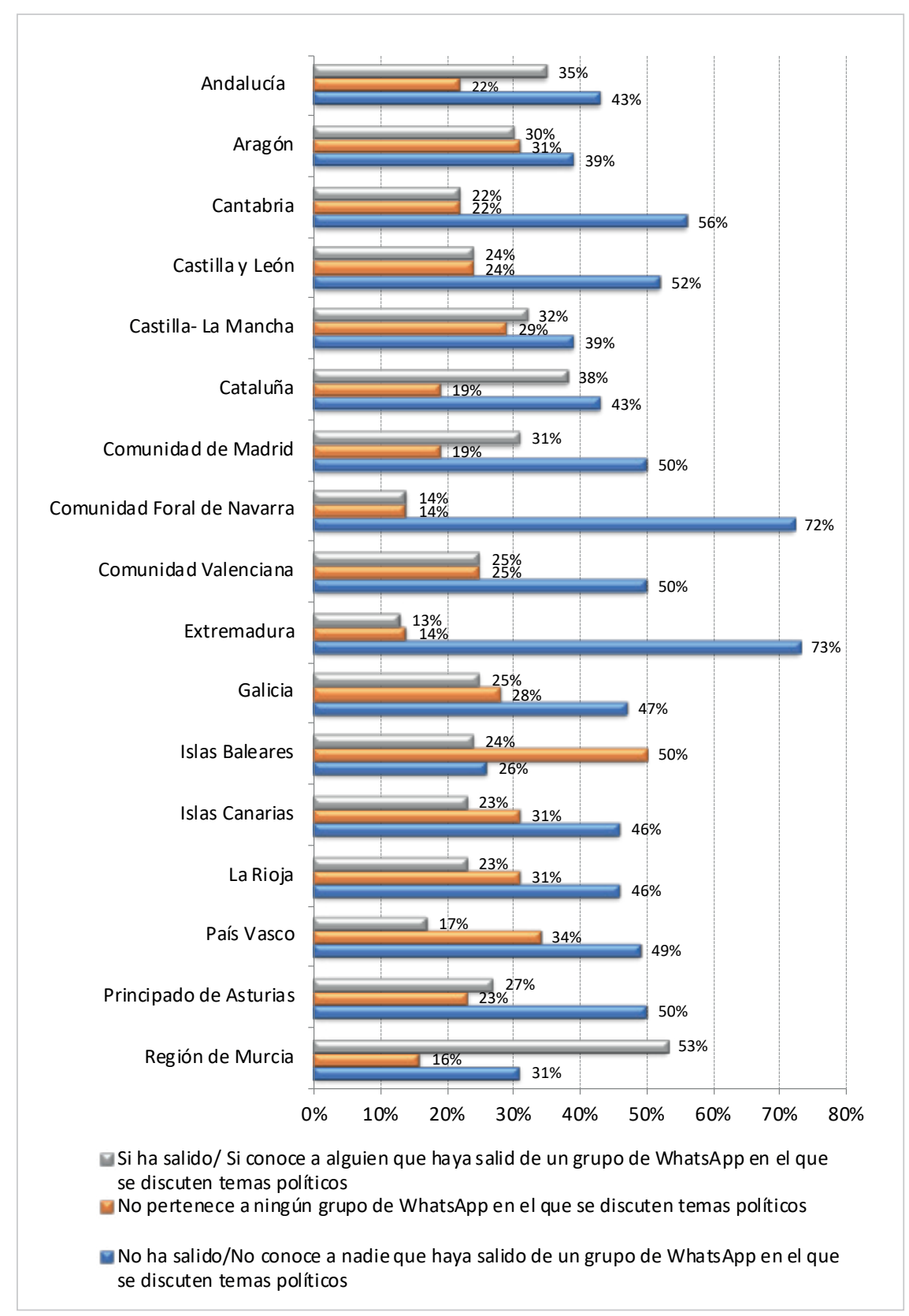

Gráfico 7. Grupos de discusión sobre actualidad política en WhatsApp, por Comunidad Autónoma 
Finalmente, hemos buscado diferencias territoriales en España sobre los grupos de WhatsApp. Se puede afirmar que hay variaciones importantes en función del lugar de residencia de los encuestados $\left(\chi^{2}[36, \mathrm{~N}=1653]\right.$ $=74,184, p=0.000$; Coeficiente de incertidumbre $=0.021$, $\mathrm{p}=0.000$ ). En Cataluña se observa que un mayor porcentaje de encuestados (38\%) declara pertenecer a un grupo que alguien (incluyéndose a sí mismos) ha abandonado por motivos políticos (sólo Murcia supera este porcentaje con el 53\%). Otras comunidades autónomas con alto abandono de grupo por cuestiones políticas son: Andalucía (35\%), Castilla-La Mancha (32\%) y Madrid (31\%).

Por otra parte, a la pregunta sobre si pertenecen a un grupo de WhatsApp en el que se discutan asuntos políticos, Extremadura (14\%); Murcia (16\%); Cataluña y Madrid (19\%) son las comunidades en comparación con el conjunto de España donde sus habitantes participan en grupos donde se habla más de política. En el lado opuesto, destaca especialmente que en las Baleares cerca del $50 \%$ de los encuestados responde no estar en un grupo de este tipo, seguidos de los ciudadanos del País Vasco (34\%) y de Canarias (31\%).

\section{Discusión y conclusiones}

Millones de ciudadanos envían mensajes de texto, fotografías y videos a diario haciendo uso de WhatsApp. Algunos de estos mensajes tienen como objetivo informar sobre la actualidad, principalmente sobre cuestiones políticas. En la investigación que aquí presentamos podemos concluir que WhatsApp incide en los hábitos informativos de los ciudadanos españoles y, en consecuencia, se ha convertido en un canal que puede contribuir a la conformación de climas de opinión. Es bueno recordar otras investigaciones que demuestran que los niveles de confianza en un medio o plataforma pueden venir determinados por la recomendación de un amigo (Turcotte et al., 2015). Esto tendría un efecto positivo en los niveles de confianza entre medio e individuo y demostraría que la comunicación interpersonal importa en la credibilidad de las noticias.

En un contexto de consolidación del llamado "espacio hipermediático", se confirma la transcendencia de WhatsApp como canal de información política si nos remitimos a los datos de esta investigación. De forma similar los estudios de Jenkins (2008); Chadwick (2013)

y Casero-Ripollés, Feenstra, y Tormey (2016) confirman esta tendencia y además demuestran cómo han cambiado los hábitos comunicativos de los ciudadanos.

A partir de nuestros datos, observamos que un $63 \%$ de los españoles recibe al menos una noticia semanal de actualidad política a través de WhatsApp, y un $24 \%$ recibe 5 o más noticias por semana. Estas cifras sirven para confirmar la primera hipótesis (a) en la que se consideraba que la plataforma era utilizada por los ciudadanos para informarse sobre la actualidad política.

El porcentaje de personas que dice utilizar WhatsApp para compartir noticias es del 77\%, una cifra que valida la hipótesis (b) de esta investigación. Finalmente, también queda demostrado, como se planteaba en la hipótesis (d), que los ciudadanos utilizan la plataforma para comentar la información política. Un 73\% de los españoles afirma que comenta la actualidad política en la aplicación. Esta conclusión complementa el estudio de Varona-Aramburu, Sánchez-Martín y Arrocha (2017) en el que se demuestra que los ciudadanos utilizan los dispositivos móviles como el primer medio de acceso a internet en España, transformando cómo el público se informa e interactúa con los contenidos informativos.

Por otra parte, se observa que WhatsApp confirma la consolidación de una sociedad orientada a la imagen. Así, el entorno de esta plataforma no escapa al predominio y prevalencia del lenguaje audiovisual en la información política. Los ciudadanos se muestran un $8 \%$ más predispuestos a compartir una información por esta plataforma si esta tiene formato vídeo o va acompañada de una fotografía, datos que confirman la hipótesis (b1) en la que se sugiere que en el entorno WhatsApp existe una mayor propensión de los ciudadanos a compartir las informaciones acompañadas de material audiovisual.

La credibilidad y confianza de la información política es un aspecto fundamental en un contexto de preocupación por los posibles efectos de las llamadas fake news en la configuración de la opinión pública, como ya señalan Bernal-Triviño y Clares-Gavilán (2019). En este sentido, hemos observado que los ciudadanos españoles otorgan a WhatsApp una credibilidad media-baja en contraste con medios como la televisión, la prensa digital o plataformas como Facebook, tal y como demostró el estudio de Pont et al. (2019). En una escala de 1 a 5, en que 1 significa "ninguna credibilidad" y 5 "total credibilidad", los españoles se sitúan de media en el 2,59 (desviación típica: 1,001). Este dato no permite validar la hipótesis (c) de este trabajo y nos remite a otras investigaciones como la de Ardèvol-Abreu y Gil de Zúñiga, (2017) que demuestran que la confianza en los medios no tiene por qué responder a su tipo: tradicionales o mainstream, ciudadanos o de redes sociales. Sin embargo, las dudas sobre la confianza o credibilidad de los ciudadanos en
Las personas mayores tienden a recibir más noticias que los más jóvenes 
este servicio de mensajería instantánea no impiden, como se ha visto, que los encuestados dejen de compartir las informaciones que les llegan por WhatsApp, hecho que puede contribuir a la viralización de noticias falsas, tal y como aseguran Bernal-Triviño y Clares-Gavilán, (2019). En cuanto a la edad de las personas tampoco se aprecia diferencias importantes a la hora de dar más o menos credibilidad a las noticias recibidas mediante este servicio de mensajería y por tanto no se puede validar la hipótesis (c1) en la que se afirma que los ciudadanos más jóvenes tienden a otorgar más credibilidad a las informaciones que reciben a través de WhatsApp.

La mensajería instantánea es un medio relevante para la discusión política y puede contribuir y reforzar la polarización política si nos atenemos a los resultados que arroja esta investigación, donde se evidencia que un tercio de los españoles ha abandonado un grupo de WhatsApp por motivaciones políticas. Si comparamos algunos de los temas recientes de agenda política española como el avance de la extrema derecha en las elecciones del 28 de abril de 2019 (28A) y del 10 de noviembre del 2019 (10N) al Congreso de los Diputados, o el denominado Procés soberanista catalán, se observa que estos temas coinciden con las zonas donde el porcentaje de abandono de grupos de WhatsApp es más alto. En Cataluña un mayor porcentaje de encuestados (38\%) afirma pertenecer a un grupo de WhatsApp que alguien (incluyéndose a sí mismos) ha abandonado por motivos políticos. Supera este porcentaje los datos de la Región de Murcia con el 53\%. La polarización podría estar detrás de estos datos si atendemos a las fechas preelectorales en las que se hizo el estudio, justo antes de las elecciones generales, municipales y autonómicas de 2019. Puede servir de ejemplo Murcia, región en la que en las elecciones del $10 \mathrm{~N}$ la formación de ultraderecha Vox ganó con 199.440 votos y tres escaños. En las anteriores elecciones (28A) había sido cuarta fuerza con 143.010 votos. Otras comunidades autónomas con alto abandono de grupo por cuestiones políticas son: Andalucía (35\%), Castilla-La Mancha (32\%) y Comunidad de Madrid (31\%). Coincide también con las comunidades en las que los ciudadanos responden en mayor medida que pertenecen a un grupo en el que se discuten asuntos políticos, Extremadura (14\%), Murcia (16\%), y Cataluña y Comunidad de Madrid (19\%). En cambio, observamos que en Baleares cerca del $50 \%$ de los encuestados responde no estar en un grupo donde se discuten cuestiones políticas, seguidos de los ciudadanos del País Vasco (34\%) y de los de Canarias (31\%). Los datos del País Vasco parecen interesantes al compararlos con otras investigaciones de zonas con alta polarización política en el pasado. Según un estudio de Valeriani y Vaccari (2018) hay una asociación clara entre la autocensura en redes sociales y la probabilidad de publicar contenidos políticos en mensajería instantánea. Los autores demostraron que la correlación era más fuerte para las personas que viven en la antigua Alemania Oriental donde, por razones históricas, grandes segmentos de la población son reacios a hablar de política en público. La correlación entre la polarización política y el abandono de grupos de WhatsApp abre un nuevo campo de estudio interesante para futuras investigaciones.

El volumen de informaciones recibidas a través de WhatsApp es un factor predictor e influye en los hábitos comunicativos de los ciudadanos en esta plataforma, dado que el número de informaciones políticas recibidas se asocia positivamente con la frecuencia con la que se comparten estas informaciones. Un hallazgo remarcable que se deriva de la presente investigación es que una alta recepción de noticias políticas indica una mayor propensión a comentar la actualidad política.

Finalmente, los datos arrojan que el género resulta un factor importante que incide en las prácticas comunicativas de los ciudadanos en WhatsApp. Una aportación destacable, especialmente para los estudios de perspectiva de género y uso de mensajería instantánea es que las mujeres tienden a recibir menos informaciones políticas a través de Whats $A p p$ que los hombres, y también tienden a comentar menos cuestiones políticas. Del mismo modo, hay menos mujeres que pertenezcan a grupos de WhatsApp en los que se habla de temas políticos y/o en los que alguien haya abandonado por motivos políticos.

La investigación muestra que la mensajería instantánea se ha instalado de forma clara en el ecosistema comunicativo en general y en el de información política en particular. Este conocimiento es importante para una comprensión global de las dinámicas de información social. Además, esta investigación aporta un desarrollo metodológico que puede ser extendido y aplicado a futuras investigaciones, así como puede ser utilizado por otros grupos de investigación.

\section{Referencias}

Ardèvol-Abreu, Alberto; Gil de Zúñiga, Homero (2017). "Effects of editorial media bias perception and media trust on the use of traditional, citizen, and social media news". Journalism \& mass communication quarterly, v. 94, n. 3, pp. 703724.

https://doi.org/10.1177/1077699016654684
La mensajería instantánea se ha instalado de forma clara en el ecosistema comunicativo en general y en el de información política en particular 
Bernal-Triviño, Ana; Clares-Gavilán, Judith (2019). “Uso del móvil y las redes sociales como canales de verificación de fake news. El caso de Maldita.es". El profesional de la información, v. 28, n. 3, e280312.

https://doi.org/10.3145/epi.2019.may.12

Besalú, Reinald; Pont-Sorribes, Carles; Sánchez-Meza, Metzeri; Castelo-Heymann, Santiago; Rovira-Sebastià, Edgar (2019). El uso de WhatApp como herramienta de información política. Barcelona: Cátedra Ideograma-UPF de Comunicación Política y Democracia.

https://cutt.ly/XhhRdBg

Boczek, Karin; Koppers, Lars (2020). "What's new about WhatsApp for news? A mixed method study on news outlets' strategies for using WhatsApp". Digital journalism, v. 8, n. 1, pp. 126-144.

https://doi.org/10.1080/21670811.2019.1692685

Calero-Vaquera, María-Luisa (2014). "El discurso del WhatsApp: entre el Messenger y el SMS”. Oralia, n. 17, pp. 85-114.

Casero-Ripollés, Andreu (2018). "Research on political information and social media: key points and challenges for the future". El profesional de la información, v. 27, n. 5, pp. 964-974.

https://doi.org/10.3145/epi.2018.sep.01

Casero-Ripollés, Andreu; Feenstra, Ramón A.; Tormey, Simon (2016). "Old and new media logics in an electoral campaign: The case of Podemos and the two-way street mediatization of politics". The international journal of press/politics, v. 21, n. 3, pp. 378-397.

http://repositori.uji.es/xmlui/handle/10234/161683

https://doi.org/10.1177/1940161216645340

Chadwick, Andrew (2013). The hybrid media system. New York: Oxford University Press. ISBN: 9780190696733

Dodds, Tomás (2019). "Reporting with WhatsApp: Mobile chat applications' impact on journalistic practices". Digital journalism, v. 7, n. 6, pp. 725-745.

https://doi.org/10.1080/21670811.2019.1592693

Elishar-Malka, Vered; Ariel, Yaron; Avidar, Ruth (2015). “Fighting, worrying and sharing: Operation 'Protective Edge' as the first WhatsApp war". Media, war \& conflict, v. 8, n. 3, pp. 329-344.

https://doi.org/10.1177/1750635215611610

Fares, Fátima (2018). "WhatsApp y periodismo. Análisis del uso de WhatsApp en los medios de información españoles". Hipertext. net: Revista académica sobre documentación digital y comunicación interactiva, n. 16, pp. 78-92.

https://doi.org/10.31009/hipertext.net.2018.i16.12

Fletcher, Richard; Park, Sora (2017). "The impact of trust in the news media on online news consumption and participation". Digital journalism, v. 5, n. 10, pp. 1281-1299.

https://doi.org/10.1080/21670811.2017.1279979

Gil de Zúñiga, Homero; Ardèvol-Abreu, Alberto; Casero-Ripollés, Andreu (2019). "WhatsApp political discussion, conventional participation and activism: exploring direct, indirect and generational effects". Information, communication \& society.

https://doi.org/10.1080/1369118X.2019.1642933

Giraldo-Giraldo, Camilo; Ríos-Londoño, Dora-Miryam; Cardona-Cifuentes, Fabio-Andrés (2018). "La gramática al margen de la norma: la escritura en WhatsApp". Lenguaje, v. 46, n. 2, pp. 311-333.

https://doi.org/10.25100/lenguaje.v46i2.6585

Go, Eun; You, Kyung-Han; Jung, Eunhwa; Shim, Hongjin (2016). "Why do we use different types of websites and assign them different levels of credibility? Structural relations among users' motives, types of websites, information credibility, and trust in the press". Computers in human behavior, v. 54, pp. 231-239.

https://doi.org/10.1016/j.chb.2015.07.046

Gutiérrez-Rubí, Antoni (2015). La política en tiempos de WhatsApp. Madrid: El País Libros.

Jenkins, Henry (2008). Convergence culture. La cultura de la convergencia de los medios de comunicación. Barcelona: Paidós. ISBN: 9788449321535

Newman, Nic; Fletcher, Richard; Schulz, Anne; Andi, Simge; Nielsen, Rasmus-Kleis (2020). Digital news report 2020. Reuters Institute, Oxford University.

https://reutersinstitute.politics.ox.ac.uk/sites/default/files/2020-06/DNR_2020_FINAL.pdf

Palomo, Bella; Sedano, Jon (2018). "WhatsApp como herramienta de verificación de fake news. El caso de B de Bulo". Revista latina de comunicación social, n. 73, pp. 1384-1397.

https://doi.org/10.4185/RLCS-2018-1312 
Pont-Sorribes, Carles; Besalú, Reinald; Rovira-Sebastià, Edgar; Castelo-Heymann, Santiago; Sánchez-Meza, Metzeri (2019). Análisis de la credibilidad de la información política en el entorno digital (Facebook, WhatsApp, prensa digital y televisión). Barcelona: Cátedra Ideograma-UPF de Comunicación Política y Democracia.

https://cutt.ly/GhhY96a

Quiroz-Pacheco, Yulvitz-Ramón (2015). “Aplicación del WhatsApp en el periodismo digital y ciudadano”. Hamut'ay, v. 2, n. 1, pp. 71-83.

https://dialnet.unirioja.es/servlet/articulo?codigo $=5665698$

Resende, Gustavo; Melo, Philipe; Sousa, Hugo; Messias, Johnnatan; Vasconcelos, Marisa A.; Almeida, Jussara M.; Benevenuto, Fabrício (2019). "(Mis) information dissemination in WhatsApp: Gathering, analyzing and countermeasures". In: The World Wide Web conference, ACM, pp. 818-828.

https://doi.org/10.1145/3308558.3313688

Sedano, Jon; Palomo, Bella (2018). “Aproximación metodológica al impacto de WhatsApp y Telegram en las redacciones". Hipertext.net: Revista académica sobre documentación digital y comunicación interactiva, n. 16, pp. 61-67.

https://doi.org/10.31009/hipertext.net.2018.i16.10

Swart, Joëlle; Peters, Chris; Broersma, Marcel (2019). "Sharing and discussing news in private social media groups: The social function of news and current affairs in location based, work-oriented and leisure-focused communities". Digital journalism, v. 7, n. 2, pp. 187-205.

https://doi.org/10.1080/21670811.2018.1465351

Tsfati, Yariv (2010). "Online news exposure and trust in the mainstream media: Exploring possible associations". American behavioral scientist, v. 54, n. 1, pp. 22-42.

https://doi.org/10.1177/0002764210376309

Turcotte, Jason; York, Chance; Irving, Jacob; Scholl, Rosanne M.; Pingree, Raymond J. (2015). "News recommendations from social media opinion leaders: Effects on media trust and information seeking". Journal of computer-mediated communication, v. 20, n. 5, pp. 520-535.

https://doi.org/10.1111/jcc4.12127

Vaccari, Cristian; Valeriani, Augusto (2018). "Dual screening, public service broadcasting, and political participation in eight Western democracies". The international journal of press/politics, v. 23, n. 3, pp. 367-388.

https://doi.org/10.1177/1940161218779170

Valenzuela, Sebastián; Bachmann, Ingrid; Bargsted, Matías (2019). "The personal is the political? What do WhatsApp users share and how it matters for news knowledge, polarization and participation in Chile". Digital journalism.

https://doi.org/10.1080/21670811.2019.1693904

Valeriani, Augusto; Vaccari, Cristian (2018). "Political talk on mobile instant messaging services: a comparative analysis of Germany, Italy, and the UK". Information, communication \& society, v. 21, n. 11, pp. 1715-1731.

https://doi.org/10.1080/1369118X.2017.1350730

Varona-Aramburu, David; Sánchez-Martín, Milagrosa; Arrocha, Roberto (2017). "Consumo de información política en dispositivos móviles en España: caracterización del usuario tipo y su interacción con las noticias". El profesional de la información, v. 26, n. 4, pp. 641-648.

https://doi.org/10.3145/epi.2017.jul.08

Yan, Wenjie; Sivakumar, Gayathri; Xenos, Michael A. (2018). "It's not cricket: examining political discussion in nonpolitical online space". Information, communication \& society, v. 21, n. 11, pp. 1571-1587.

https://doi.org/10.1080/1369118X.2017.1340499 